\title{
Schwere Sepsis und septischer Schock auf deutschen Intensivstationen
}

SepNet Critical Care Trials Group. Incidence of severe sepsis and septic shock in German intensive care units: the prospective, multicentre INSEP study. Intensive Care Med 2016; 42: 1980 - 1989

Die hohe Sepsis-assoziierte Mortalität und Morbidität verlangen gute epidemiologische Daten, um klinische Entwicklungen und Forschung steuern und bewerten zu können. Ebenso wichtig sind länderspezifische Werte zur internationalen Vergleichbarkeit. Dafür haben die Forscher der SepNet Clinical Trials Group eine große Patientenpopulation auf deutschen Intensivstationen untersucht.

Zum jetzigen Zeitpunkt ist Sepsis weiterhin ein häufiges Krankheitsbild mit hoher assoziierter Mortalität auf deutschen Intensivstationen. Dies haben die Mitglieder der SepNet Clinical Trials Group 10 Jahre nach der ersten Untersuchung ähnlicher Art in einer weiteren Studie herausgearbeitet. In einer prospektiven, multizentrischen, longitudinalen Beobachtungsstudie an 133 Intensivstationen in Deutschland schlossen sie zwischen dem 4.November und 1.Dezember 201311883 Patienten ein. Die Outcome-Daten dieser Patienten verfolgten die Autoren bis zum 2. Februar 2014. Für die Untersuchung wurden Sepsis und septischer Schock anhand der SEPSIS-1Kriterien definiert, wobei die Autoren ebenfalls einen Vergleich mit den SEPSIS-3-Kriterien durchführten.

Innerhalb des Patientenkollektivs diagnostizierten sie nach den Sepsis-1-Kriterien bei 218 Patienten eine schwere Sepsis (Intensiv-Mortalität 16,7\%, Krankenhaus-Mortalität 34,4\%) und bei 1285 Fällen einen septischen Schock (IntensivMortalität 37,3\%, Krankenhaus-Mortalität 43,3\%; gesamt 1503; 12,6\%). Anhand Sepsis-3-Kriterien wäre bei nur 848 Patienten ein septischer Schock diagnostiziert worden (Intensiv-Mortalität 44,3\%, Krankenhaus-Mortalität 50,9\%).

Die Punktprävalenz von Patienten mit Sepsis oder septischem Schock am 4.11.2013 betrug 17,9\% (95\% Cl 16,319,7) in der Gesamtpopulation ( 345 aus 1924 Patienten) und reichte von $16,0 \%$
$(95 \% \mathrm{Cl} 13,9-18,4)$ bis $23,8 \%(95 \% \mathrm{Cl}$ 19,0-29,4) in Abhängigkeit des Krankenhaustyps (universitär, kommunal, etc.) Die Intensiv-Sterblichkeit aller Patienten ohne Sepsis betrug 6\% und die Krankenhaus-Sterblichkeit 9,6\%.

Die Inzidenz von schwerer Sepsis und septischem Schock betrug 11,64 (95\% $\mathrm{Cl} 10,51$-12,86) pro 1000 Intensivtagen (388 neue Fälle in 33,333 Patienten-Tagen).

Die Forscher beobachteten u.a. weitere Punkte:

- 57,2\% der für die Sepsis ursächlichen Infektionen waren nosokomialen Ursprungs

- ca. die Hälfte dieser wurde auf Intensivstation erworben

- Infektions-Gebiete: 46,6\% untere Atemwege, 28,7\% Abdomen, 12,6\% Urogenitaltrakt

- Pilze fanden sich in 9,7\% der Blutkulturen und in 19,3\% der am Infektionsort gewonnenen Kulturen

- positive Blutkulturen fanden sich in 29,9\% der Fälle

- die häufigsten SIRS Kriterien waren Tachypnoe, Tachykardie und Leukozytose/-penie $(81,0,80,9$, und $76,7 \%)$.

\section{FAZIT}

In Zusammenschau stellt die SepNetGruppe um Gernot Marx dar, dass Sepsis weiterhin eine häufige und lebensbedrohliche Erkrankung auf deutschen Intensivstationen mit hohem Anteil an nosokomialen Infektionen ist. Die neuen SEPSIS-3-Kriterien identifizieren eine schwerer erkrankte Patientengruppe mit höherer Mortalität.

Dr. med. Johannes Achenbach, Hannover

\section{Kommentar}

Die INSEP-Studie ist eine nationale Erfassung der Inzidenz von Sepsis und septischem Schock auf deutschen Intensivstationen während eines 4-wöchigen Untersuchungszeitraums. Die Ergebnisse verwundern nicht. Die Sepsis bleibt eine häufige Erkrankung auf der Intensivstation mit einer hohen ITS- bzw. Krankenhaussterblichkeit. Diese ist mit anderen europäischen Ländern vergleichbar. Auch eine Zunahme an Gram-negativen Erregern wurde bereits durch andere Untersucher gezeigt. Ähnliches gilt für die hohe Anzahl an nosokomialen Infektionen [1].

Interessant, aber auch schwierig wird der Vergleich mit anderen Studien, wenn die SEPSIS-3-Definitionen herangezogen werden. Dies wird auch zukünftig ein Problem sein, wenn Vergleiche mit älteren epidemiologischen Studien gezogen werden. Die Ergebnisse der INSEP-Studie zeigen, dass durch Verwendung der SEPSIS-3-Definitionen die Sterblichkeit auf der Intensivstation bei Patienten mit septischem Schock im Vergleich zur SEPSIS-1-Definition deutlich ansteigt (37,3 vs. 44,3\%). Dies spiegelt die Ergebnisse von Kaukonen et al. [2] wieder, die zeigen konnten, dass durch die Verwendung der SEPSIS-1-Definitionen einer von 8 Patienten mit schwerer Sepsis, Organversagen und hoher Sterblichkeit nicht berücksichtigt wird. Daher war eine neue Definition dringend notwendig, auch wenn es weiterhin viel Aufklärungs- und Überzeugungsarbeit bedeutet, diese Änderungen in der Fläche zu erreichen. Wahrscheinlich muss man das Jahr 2016 als Zäsur in der epidemiologischen Sepsisforschung ansehen.

\section{Ausblick}

Die Ergebnisse der INSEP-Studie geben uns aber noch weitere Aufträge: Wir müssen die bisher gewonnenen Erkenntnisse nutzen und sie konsequenter um- 
setzen. Zum Beispiel müsste die SepsisFrüherkennung weiter ausgebaut werden, damit die Diagnosestellung und Therapie nicht erst auf der Intensivstation erfolgt. Vielleicht hilft dabei auch die Verwendung des qSOFA-Scores. Wir müssen auch die bisher identifizierten/ beschlossenen Sepsisbündel konsequenter anwenden bzw. deren Anwendung überwachen. Des Weiteren muss weiter Ursachenforschung betrieben werden, um die hohe Rate an nosokomialen Infektionen wirksam zu bekämpfen. Auf diesem Feld ist ein enormes Potential für eine Therapieoptimierung mit dem Ziel der Verringerung der Sterblichkeit bei septischen Patienten erkennbar.
Literatur

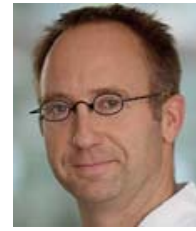

Prof. Dr. med. Jan P. Roesner, Klinik für

Anästhesie und Intensivmedizin, Klinikum Südstadt Rostock
[1] Kaye KS, Pogue JM. Infections Caused by Resistant Gram-Negative Bacteria: Epidemiology and Management. Pharmacotherapy 2015; 35: 949-962

[2] Kaukonen KM et al. Systemic Inflammatory Response Syndrome Criteria in Defining Severe Sepsis N Engl J Med 2015; 372: 1629-1638 